\title{
Enhancement of salivary $\lg A$ response to a DNA vaccine against Streptococcus mutans wall- associated protein $A$ in mice by plasmid-based adjuvants
}

\author{
Thomas K. Hant and My Lien Dao \\ Department of Biology, University of South Florida, Tampa, FL, USA
}

Correspondence

My Lien Dao

mdao@cas.usf.edu

Received 22 October 2006

Accepted 8 January 2007

\begin{abstract}
A specific salivary $\lg A(\lg A)$ response was obtained in mice by intranasal immunization with a naked DNA vaccine consisting of the Streptococcus mutans wall-associated protein A gene $(w a p A)$ inserted into the mammalian expression vector pcDNA3.1/V5/His-TOPO. In the present study, the vaccine, referred to as pcDNA-wapA, was administered with or without the cationic lipid DMRIE-C. No mucosal response was observed in mice immunized with the vaccine alone, whereas a weak and temporal slgA response was obtained when the vaccine was mixed with DMRIE-C. To investigate the use of pcDNA containing the interleukin 5 (IL-5) gene (pcDNA-il-5) or the cholera toxin B gene (pcDNA-ctb) as genetic adjuvants, these constructs were used in co-immunization studies. The enhancement effect was transient with pcDNA-il-5, but longer lasting with pcDNA-ctb, thus supporting the use of the latter as a genetic adjuvant to DNA vaccine.
\end{abstract}

\section{INTRODUCTION}

Dental caries is a widespread infectious disease, of which the principal causative agent is Streptococcus mutans. In recent years, caries prevalence and distribution has shifted from circulating evenly among the population to disproportionately affecting individuals in economically challenged populations worldwide (Curzon \& Preston, 2004; Rugg-gunn, 2001). Hence, the production of a safe and cost-effective dental caries vaccine to block tooth colonization and plaque build up by $S$. mutans has been a high priority in dental research. With recent advances in DNA vaccine technology comes the hope for a more economical way to immunize against infectious diseases. DNA vaccine is advantageous when considering the ease of isolation of plasmid DNA, low cost for mass-production, stability to extreme temperatures and ease of administration (Lowe et al., 2006). The idea of a DNA dental caries vaccine is attractive, but the full potential of DNA vaccines has not yet been fully realized, and conditions conducive to a longlasting mucosal salivary IgA ( $\operatorname{IgA})$ immune response have not yet been established.

To enhance the desired mucosal immune response, a number of adjuvants have been used for protein- and

tPresent address: Department of Cancer Immunology and AIDS, DanaFarber Cancer Institute, Harvard Medical School, and Department of Medicine, Harvard Medical School, Boston MA, USA.

Abbreviations: GST, glutathione S-transferase; HRP, horseradish peroxidase; IL, interleukin; slgA, salivary lgA.
DNA-based vaccines. The adjuvants used in protein or peptide vaccines include cholera toxin, cholera toxin B subunit (CTB) and a detoxified heat labile Escherichia coli toxin (LT R292G) (De Magistris, et al., 1998; Wu \& Russell, 1993), and DNA vaccine adjuvants include liposome and various cationic lipids (Klavinskis et al., 1997, 1999; Ochiya et al., 1999; Perrie et al., 2001). Cytokines were found to further provide immunopotentiating effects, for instance interleukin (IL)- 4 or IL-10 induced a Th2 type response, whereas IL-12 and/or a granulocyte/macrophage colony stimulating factor favoured a Th1 type of response (Donnelly et al., 1997; Okada et al., 1997; Ramsay \& Kohonen-Corish, 1993; Whittle et al., 1997). Intranasal administration of recombinant adenovirus vectors expressing IL-5 has been shown to enhance mucosal IgA response to co-expressed heterologous antigen in the lung of immunized mice (Ramsay \& Kohonen-Corish, 1993). Increased IgA response to Salmonella lipopolysaccharide has also been observed in mice immunized with a bacterial strain engineered to express murine IL-5 (Whiley et al., 1988).

The goal of the present study was to explore the ability of plasmids encoding CTB or cytokine IL-5 as a genetic adjuvant to boost the mucosal antibody response to a DNA vaccine administered intranasally. The target antigen used in this study was the S. mutans wall-associated protein A (WapA), a factor involved in tooth colonization and buildup of dental plaque (Qian \& Dao, 1993). Three different plasmids were constructed by separately cloning wapA, il-5 and $c t b$ into the mammalian expression vector pcDNA3.1/ 
V5-His-TOPO. The constructs, designated pcDNA-wapA, pcDNA-il-5 and pcDNA-ctb, were mixed with the cationic lipid transfection adjuvant DMRIE-C, used to transfect HeLa cells in order to check for the expression of the corresponding proteins in these cells, and then administered intranasally to the BALB/C mice. Specific SIgA was determined for comparison between pcDNA-wapA without DMRIE-C, with DMRIE-C, with DMRIE-C plus pcDNA-il-5 or with DMRIE-C plus pcDNA-ctb.

\section{METHODS}

Experimental animals. BALB/c female mice of 6-8 weeks old were purchased from Charles River Laboratories and were maintained in the animal facilities of the University of South Florida, College of Medicine. All protocols using mice were approved by the Institutional Animal Care and Use Committee at the University of South Florida. The University programme and facilities for animal care and use are fully accredited by the Association for Assessment and Accreditation of Laboratory Animal Care International (AAALAC).

DNA vaccine construction. The three clones needed for this study consisted of a recombinant E. coli TOP 10 clone containing the wapA, $i l-5$ or $c t b$ gene inserted separately into the mammalian expression vector pcDNA3.1/V5/His-TOPO (Invitrogen) (Fig. 1a). In order to improve the translation efficiency of the expressed genes in mammalian hosts, the Kozak sequence ACCATG was incorporated into all the inserted genes. The final constructs obtained were designated pcDNA-wapA, pcDNA-il-5 and pcDNA-ctb. The recombinant expression plasmid pcDNA-wapA was constructed as described previously (Han et al., 2001), whereas, pcDNA-il-5 and pcDNA- $c t b$ were cloned in the present study as follows: recombinant
E. coli HB101 containing IL-5 cDNA cloned into the vector pBR322 was obtained from the American Type Culture Collection (ATCC) and the plasmid was used as a template for PCR amplification of il-5 using primers that were designed based on the IL-5 DNA sequence available in GenBank (accession no. NM_010558). The primers used included the forward primer, 5'-ACC ATG AGA AGG ATG CTT CTG CAC-3', and the reverse primer, 5' -TCA GCC TTC CAT TGC CCA-3'. The resulting PCR product was cloned downstream of the CMV promoter into the pcDNA3.1/V5/His-TOPO vector. Construction of a plasmid containing the $c t b$ gene was performed similarly using genomic DNA of Vibrio cholerae (ATCC) as a template for the PCR amplification of the $c t b$ gene. Primers for the amplification of $c t b$ were designed based on the gene sequence available in GenBank (accession no. D30053), and included the forward primer, 5'-ACC ATG ACA CCT CAA AAT ATT ACT GAT T- $3^{\prime}$, and the reverse primer, $5^{\prime}$-TTA ATT TGC CAT ACT AAT TGC GG-3'.

DNA sequencing. The plasmids constructed as described above were purified to sequencing grade by a modified alkaline lysis method using the HiSpeed plasmid maxi kit (Qiagen) and following the manufacturer's instruction. Sequences were determined at the DNA sequencing core facility of the H. Lee Moffitt Cancer Center and Research Institute, Tampa, FL, USA, in order to confirm the sequence and orientation of the target genes. The primer sets for sequencing were the T7 forward primer, 5' -TAA TAC GAC TCA CTA TAG GG-3', and the BGH reverse primer, 5'-TAG AAG GCA CAG TCG AGG-3'.

Expression and purification of WapA from E. coli recombinant clones. To obtain enough protein for the preparation of rabbit antibodies and immunochemical analysis of WapA in the present study, purification of WapA protein was optimized and scaled up. WapA was obtained from the corresponding recombinant E. coli BL21 (DE3) clone as a fusion protein with glutathione S-transferase (GST) (Yoder et al., 2000). The clone was cultured at $37{ }^{\circ} \mathrm{C}$ in an incubator shaker overnight in batches of $500 \mathrm{ml} 2 \times \mathrm{YT}$ medium (prepared by (a)

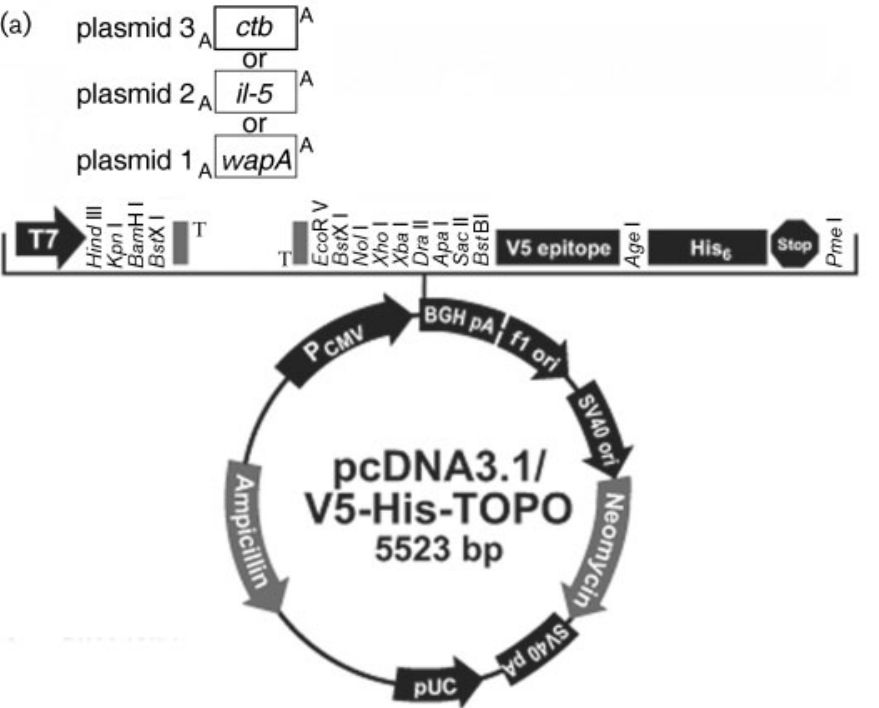

(b)

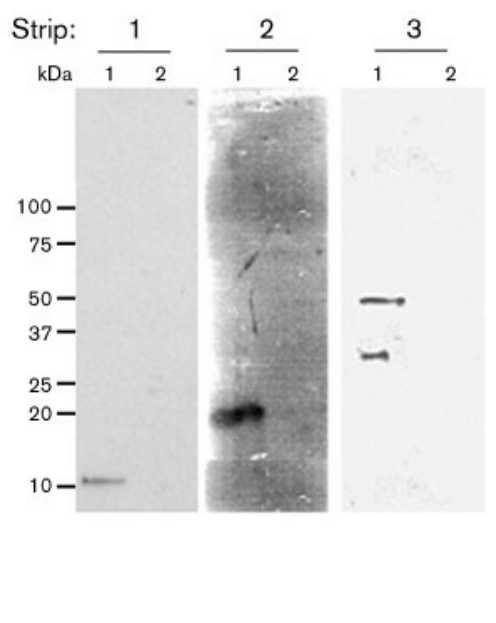

Fig. 1. Cloning of $c t b, i l-5$ and $w a p A$ into pcDNA3.1/V5/His-TOPO and in vitro expression of the cloned genes. (a) The diagram shows the site at which the target genes were inserted separately into the vector. Copyright 2007 Invitrogen Corporation, used with permission. (b) Western immunoblotting of transfected HeLa cells shows the expression of antigens with the anticipated molecular size: from left to right, strip 1/lane 1, CTB; strip 2/lane 1, IL-5; strip 3/lane 1, WapA with a smaller band of approximately $29 \mathrm{kDa}$ corresponding to the antigen $\mathrm{A}(\mathrm{AgA})$ protein, which was derived from WapA by proteolytic cleavage. In all three strips, lane 2 containing HeLa cells transfected with empty vector was negative. 
dissolving $16 \mathrm{~g}$ tryptone, $10 \mathrm{~g}$ yeast extract and $5 \mathrm{~g} \mathrm{NaCl}$ in $900 \mathrm{ml}$ distilled $\mathrm{H}_{2} \mathrm{O}, \mathrm{pH}$ 7.0) supplemented with $100 \mu \mathrm{g}$ ampicillin $\mathrm{ml}^{-1}$ $(2 \times$ YTA $)$. The following day, the overnight culture was diluted 1:100 into fresh pre-warmed $2 \times$ YTA medium, and incubation was continued at $37^{\circ} \mathrm{C}$ until the culture reached an $\mathrm{OD}_{600}$ of 1 . Induction of protein expression and preparation of the protein lysate were performed as described previously (Yoder et al., 2000). Purification of GST-WapA was carried out by adding $50 \%(\mathrm{v} / \mathrm{v})$ slurry of glutathione-linked Sepharose 4B (Pharmacia) to the protein lysate, and the mixture was incubated on a rocker for $30 \mathrm{~min}$ at room temperature. The mixture was transferred onto a chromatography column, followed by washing with $50 \mathrm{ml}$ PBS containing $1 \mathrm{mM}$ PMSF. The fusion protein was eluted by $10 \mathrm{mM}$ reduced glutathione in five aliquots of $300 \mu \mathrm{l}$ each. Protein concentration was determined by optical density reading at $280 \mathrm{~nm}$. One $\mathrm{OD}_{280}$ equals $0.5 \mathrm{mg}$ protein $\mathrm{ml}^{-1}$ using a formula based on the absorption coefficient of free GST. Protein purity was analysed by SDS-PAGE using $12 \%(\mathrm{w} / \mathrm{v})$ acrylamide gel.

Immunogenicity of GST-WapA fusion protein. Considering the advantage of skipping the cleavage of GST from the fusion protein in term of time and money, this step was omitted from the production of WapA protein for immunization. The validity of this approach was tested by demonstrating the reactivity of GST-WapA with a previously prepared rabbit anti-WapA antibody.

Preparation of rabbit polyclonal antibody against WapA. Rabbit polyclonal antibody was prepared as follows: briefly, two $4 \mathrm{~kg}$ female albino rabbits from the New Zealand strains (Harlan) were immunized intramuscularly with $100 \mu \mathrm{g}$ purified GST-WapA fusion protein mixed in a $1: 1(\mathrm{v} / \mathrm{v})$ ratio with TiterMax Gold (Sigma) adjuvant. Two booster injections of the same vaccine construct were administered at 3 week intervals. Blood was collected before the immunization and 1 week after each booster injection. Serum was separated by centrifugation and decomplemented by heating at $55{ }^{\circ} \mathrm{C}$ in a water bath for $30 \mathrm{~min}$. Serum was sterilized by adding 1/10 (v/v) chloroform, mixed and centrifuged to obtain the aqueous upper layer containing the antibody.

Absorption of antiserum. Non-specific binding of antiserum to WapA was removed by extensive absorption of serum against E. coli, GST and HeLa cells as described previously (Han et al., 2001). For the absorption of anti-GST antibodies, glutathione Sepharose 4B attached with GST was packed onto a gravity-flow column. Serum was loaded on the column and incubated for $1 \mathrm{~h}$ at room temperature. Incubation was continued for $1 \mathrm{~h}$ at $4{ }^{\circ} \mathrm{C}$, and the absorbed serum flow-through was collected. Fractions were tested for antibody activity using a dot immunobinding assay.

Purification of IgG antibody against WapA. WapA specific antibodies from post-immune rabbit serum were purified using the Melon gel IgG purification kit (Pierce), according to the procedure specified by the manufacturer. Serum was diluted by $1: 10(\mathrm{v} / \mathrm{v})$ with Melon gel purification buffer and loaded onto a gravity-flow column. The specific antibody was eluted with the purification buffer, and absorbance of the antibody fractions was measured at $280 \mathrm{~nm}$.

Transfection. HeLa cells were purchased from ATCC and transfected separately with the plasmid constructs pcDNA-wapA without DMRIE-C, pcDNA-wapA with DMRIE-C, pcDNA-wapA with DMRIE-C and pcDNA-il-5 or pcDNA- $c t b$. DMRIE-C (Invitrogen) was added at $1: 2$ molar ratio of pcDNA construct/DMRIE-C, providing the optimal condition determined in previous studies (Han \& Dao, 2005). Briefly, HeLa cells were seeded with $2 \times 10^{5}$ cells in $2 \mathrm{ml}$ culture medium, which consisted of Dulbecco's modified Eagle's medium supplemented with $1 \mathrm{mM}$ sodium pyruvate, $5 \mathrm{mM}$ L-glutamine, $100 \mathrm{IU}$ penicillin, $100 \mu \mathrm{g}$ streptomycin $\mathrm{ml}^{-1}, 0.25 \mu \mathrm{g}$ amphotericin $\mathrm{ml}^{-1}$ and $10 \%$ fetal bovine serum (FBS), onto a 6-well tissue culture plate and grown overnight at $37^{\circ} \mathrm{C}$ in a $\mathrm{CO}_{2}$ incubator until approximately $70 \%$ confluence. The cells were washed once with $2 \mathrm{ml}$ serum-free medium, and then overlaid with the lipid-DNA complex solution. After $4 \mathrm{~h}$ of incubation, growth medium containing $20 \%$ FBS was added to each well, and the incubation was continued for another $24 \mathrm{~h}$. At $24 \mathrm{~h}$ post-transfection, the cells were solubilized with M-PER mammalian protein extraction reagent (Pierce), and the cell suspension was collected.

Western immunoblotting of transfected HeLa cells. Expression of the transfected genes was assayed by Western blotting, as previously described (Han et al., 2001). Briefly, a sonic extract of the transfected HeLa cells was separated by SDS-PAGE on a $12 \%$ gel and transferred by electrophoresis onto a piece of nitrocellulose membrane, which was then blocked by incubation with PBS containing 5\% skimmed-milk powder and then probed with 1:500 dilution of monoclonal anti-IL-5 (Imgenex) or anti-CTB (Biodesign) antibody, or absorbed and purified polyclonal anti-WapA antibody raised as described previously. The blot was incubated with a horseradish peroxidase (HRP)-conjugated corresponding secondary antibody diluted to $1: 2000$, and specific bands were developed on an X-ray film (Kodak) using an HRP chemiluminescent substrate (Amersham).

Immunization protocols. For DNA vaccination, $50 \mu \mathrm{g}$ each DNA vaccine constructed was intranasally administered in the presence of DMRIE-C at 1:2 molar ratio of DNA/DMRIE-C unless otherwise indicated. The mice were immunized, six mice per group, as follows: group 1 with pcDNA-wapA alone; group 2 with pcDNA-wapA plus pcDNA-il-5; group 3 with pcDNA-wapA plus pcDNA-ctb; group 4 with pcDNA-wapA without DMRIE-C. All mice were immunized four times biweekly.

Collection of saliva. Mice were injected intraperitoneally with $100 \mu \mathrm{l}$ pilocarpin $\left(1 \mathrm{mg} \mathrm{ml}^{-1}\right)$ to induce salivary flow. Saliva was collected by aspiration from the cheek pouch using a plastic pipette tip. PMSF was added at $1 \mathrm{mM}$ as a protease inhibitor and the saliva was stored at $-70{ }^{\circ} \mathrm{C}$ until use, at which time the sample was centrifuged and the supernatant used in immunochemical assays.

Dot immunobinding assay. The presence of specific IgA expressed in the saliva of the immunized mice was detected by immunodot analysis as follows. A piece of 96-well-embossed nitrocellulose membrane was dotted with $10 \mu \mathrm{l}$ per dot of $5 \mu \mathrm{g}$ purified WapA protein $\mathrm{ml}^{-1}$. The membrane was blocked with $5 \%$ skimmed milk for $1 \mathrm{~h}$ and small membrane pieces containing the antigen dots were cut out and incubated separately overnight at $4{ }^{\circ} \mathrm{C}$ with diluted $(1: 2)$ saliva samples. After extensive washing, the membrane was incubated with an alkaline-phosphatase-conjugated anti-mouse IgA diluted to $1: 10000$ for $1 \mathrm{~h}$, followed by antibody detection by staining with a chromogenic substrate solution containing $\mathrm{O}$-dianisidine tetrazotized $\left(0.25 \mathrm{mg} \mathrm{ml}^{-1}\right)$ and $\beta$-naphthyl acid phosphate $\left(0.25 \mathrm{mg} \mathrm{ml}^{-1}\right)$ in $0.06 \mathrm{M}$ sodium borate buffer at $\mathrm{pH} 9.7$ (Dao, 1985). A purple colouration was indicative of the presence of sIgA against WapA. Saliva from pre-immunized mice served as negative controls.

ELISA. To follow the production of specific sIgA over time, ELISA was used to determine the sIgA titres as described previously (Han \& Dao, 2005). Serial twofold dilutions of saliva were made and then incubated at $4{ }^{\circ} \mathrm{C}$ for overnight in the purified WapA-coated plate. The level of specifically bound sIgA antibody was quantified by using an HRP-conjugated goat anti-mouse IgA diluted to $1: 1000$ and then read on a fluorometer with $325 \mathrm{~nm}$ excitation and $420 \mathrm{~nm}$ emission. Production of sIgA (mean titre from six animals and SD) was calculated and plotted as a function of time post-immunization. The end-point titres were expressed as the highest saliva dilution that 
resulted in an absorbance value three times the SD above the mean of the pre-immune saliva.

Statistical analysis. Data for saliva antibody titres were exponentially transformed and statistical differences were determined by using Kruskal-Wallis nonparametric one-way analysis of variance (ANOVA) with Dunn's multiple comparison post test.

\section{RESULTS AND DISCUSSION}

\section{Expression of DNA vaccine plasmids containing the target gene in eukaryotic cells}

The expression of the genes inserted into the plasmid vector was verified by in vitro transfection of HeLa cells. Western blotting confirmed that these cells expressed the corresponding antigens, based on the anticipated size for CTB, IL-5 or WapA (Fig. 1b). In contrast, no immunoreactive protein was expressed in the HeLa cells that were transfected either with the empty pcDNA3.1/V5/HisTOPO vector or with pcDNA-wapA without the cationic adjuvant DMRIE-C. Thus, cationic lipid was proven to be a necessary adjuvant.

\section{Antigenicity and serospecificity to GST-WapA fusion protein}

Antisera obtained from rabbits immunized with GSTWapA fusion protein were adsorbed with E. coli antigens and GST, then tested against $S$. mutans GS5 wild-type and E. coli recombinant clones by immunodot analysis. The results showed the presence of specific antibody (Fig. 2). The GST-WapA fusion protein elicited a specific antibody response to the WapA in rabbit, suggesting that the presence of GST did not interfere with the immunogenicity of the target protein. Thus, the cleavage of GST was not necessary, at least in studies involving immunization of murine models of experimental caries.

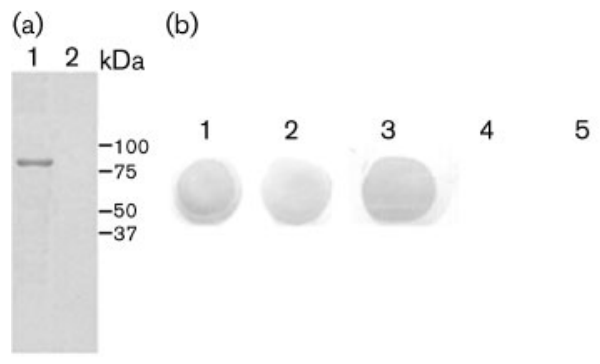

Fig. 2. Immunogenicity of GST-WapA fusion protein. (a) Lane 1 shows purified GST-WapA fusion protein and lane 2 shows purified GST. (b) Immunodot analysis of (1) S. mutans wild-type sonic extract, (2) E. coli recombinant clone harbouring wapA in pcDNA vector, (3) recombinant E. coli expressing GST-WapA fusion protein. Only (1), (2) and (3) were positive against rabbit anti-WapA antiserum. As anticipated, there was no reactivity with purified GST (4) or E. coli lysate (5).

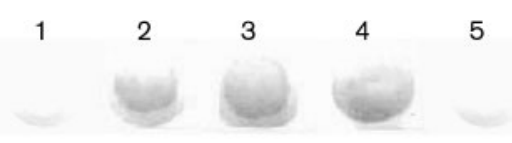

Fig. 3. Detection of specific slgA production in immunized mice. Immunodot analysis showed the presence of slgA antibody to WapA in mice immunized with (2) pcDNA-wapA with DMRIE-C, (3) pcDNA-wapA with DMRIE-C and pcDNA-il-5, and (4) pcDNAwapA with DMRIE-C and pcDNA-ctb. No immunogenicity was observed for the non-immunized mice (1) or mice immunized with pcDNA-wapA in the absence of DMRIE-C (5).

\section{Antibody response}

Two weeks after the first booster immunization, saliva samples were collected from immunized or control mice, and the presence of anti-WapA sIgA was detected by immunodot analysis. Group 1 mice (immunized with pcDNA-wapA), group 2 mice (co-immunized with pcDNA-wapA plus pcDNA-il-5) and group 3 mice (immunized with pcDNA-wapA plus pcDNA-ctb) showed the presence of anti-WapA sIgA, while no reaction was detected in pre-immune saliva (Fig. 3). No anti-WapA sIgA was produced by group 4 mice (immunized with pcDNAwapA without DMRIE-C), a finding consistent with the

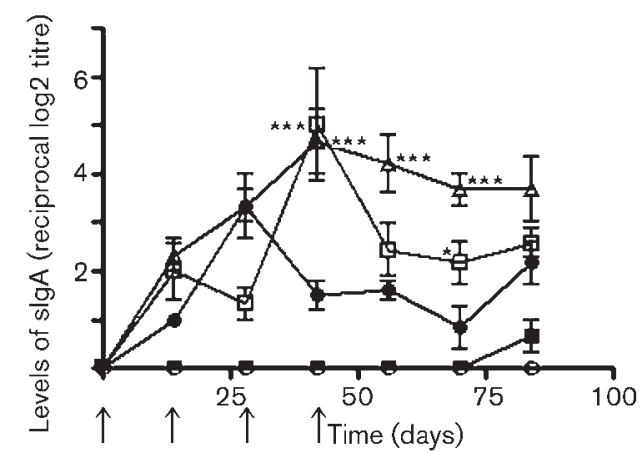

Fig. 4. Determination of the levels of slgA anti-WapA response in the immunized mice. All constructs were mixed with the cationic lipid DMRIE-C unless otherwise stated. Primary administration was at time 0 , and subsequent boosts were given biweekly ( $\uparrow$ ). Saliva was collected before each booster administration. The levels of $\operatorname{slg} A$ were expressed as the mean \pm SD of log2 antibody titres $(n=6$ mice). Mice that were immunized with pcDNA-wapA with DMRIE$\mathrm{C}$ showed an increase in slgA level after the second immunization, followed by a drop thereafter, despite two additional doses of the vaccine. Relatively higher levels were obtained in the mice immunized with pcDNA-wapA/ctb or pcDNA-wapA/il-5 after three doses of the corresponding DNA constructs. Subsequent decline in slgA levels was significantly slower in the mice receiving pcDNA-wapA/ctb. Asterisks indicate a significant difference from all other mice groups: ${ }^{\star \star \star} P<0.001 ;{ }^{\star} P<0.05$ (mean $+\mathrm{SE}$ ). $\bigcirc$, Non-immunized; ○, pcDNA-wapA; $\square$, pcDNA-wapAl-il-5; $\triangle$, pcDNA-wapAl-ctb; $\mathbf{\square}$, wapA only without DMRIE-C. 
lack of WapA expression by HeLa cells transfected with pcDNA-wapA without DMRIE-C.

\section{Immunomodulatory effect of genetic adjuvants on humoral immunity}

To investigate the influence of co-immunization with pcDNA-il-5 or pcDNA-ctb on the level and duration of sIgA response, saliva from the different groups of mice was collected and analysed at various time intervals. Group 1 mice that were immunized with pcDNA-wapA were shown to produce a significant specific sIgA antibody response $(P<0.05)$ after a primary immunization and one booster dose $(P<0.05)$, but there was no apparent memory enhancement by subsequent booster doses (Fig. 4). Group 2 and group 3 mice showed a significantly higher $\operatorname{sigA}$ response after one primary and two booster doses of the corresponding DNA constructs $(P<0.001)$. However, this was followed by a drop in the sIgA level in group 2 mice that received pcDNA-wapA and pcDNA-il-5 as an adjuvant despite an additional booster dose, although the level was still significantly higher than in group 1 mice that did not received any DNA adjuvant. Data from group 3 mice indicated that, unlike with pcDNA-il-5, the use of pcDNA$c t b$ was associated with a sustained production of sIgA levels for an additional 4 weeks $(P<0.001)$ as shown in Fig. 4. In summary, the antigen-specific sIgA response to the DNA vaccine pcDNA-wapA was generally enhanced by coimmunization with pcDNA-il-5 or pcDNA-ctb, suggesting an immunomodulatory effect of IL-5 or CTB expression. Additionally, the enhancement obtained by co-immunization with pcDNA-ctb could be attributed to the carrier function of CTB that is associated with the high affinity of CTB to GM1 ganglioside receptors present at the surface of epithelial cells and leukocytes, thus promoting the uptake and presentation of co-expressed WapA to the mucosal immune system. George-Chandy et al. (2001) reported a similar observation that the coupling of antigen to CTB augmented the antigen-presenting capacity to dendritic cells and B cells, as well as macrophages. Insufficient memory response was consistent with data from other studies with DNA vaccine (Letvin et al., 1997; Robinson et al., 1999; Shiver et al., 1996). Jia et al. (2006) showed an immunomodulatory effect of anti-caries DNA vaccine by fusion of cytotoxic T lymphocyte antigen-4 (CTLA4) to $S$. mutans antigens, suggesting that the fusion vaccine may improve the protective efficacy of DNA vaccine against dental caries.

Our work is to the best of our knowledge the first to test the usefulness of genetic adjuvants in immunization with anti-caries DNA vaccine, and the results indicate that co-immunization with pcDNA-ctb induces steadier production of the sIgA response than with pcDNA-il-5, supporting the use of this construct as an adjuvant in DNA vaccine regimens. This observation is in agreement with the recognized use of CTB as an adjuvant for the induction of a mucosal response to a protein-based vaccine in mice (Wu \& Russell, 1993). Furthermore, unlike cholera toxin and cholera toxin A subunits, CTB is not toxic, and hence is considered as a good candidate adjuvant for human use (Yasuda et al., 1998).

\section{ACKNOWLEDGEMENTS}

This work was supported in part by NIH/NIDCR public health service grants R21 DEO14926 and R21 DEO15127.

\section{REFERENCES}

Curzon, M. E. J. \& Preston, A. J. (2004). Risk groups: nursing bottle caries/caries in elderly. Caries Res 38, 24-33.

Dao, M. L. (1985). An improved method of antigen detection on nitrocellulose: in situ staining of alkaline phosphatase conjugated antibody. J Immunol Methods 82, 225-231.

De Magistris, M. T., Pizza, M., Douce, G., Ghiara, P., Dougan, G. \& Rappuoli, R. (1998). Adjuvant effect of non-toxic mutants of $E$. coli heat-labile enterotoxin following intranasal, oral and intravaginal immunization. Dev Biol Stand 92, 123-126.

Donnelly, J. J., Ulmer, J. B. \& Liu, M. A. (1997). DNA vaccines. Life Sci 60, 163-172.

George-Chandy, A., Eriksson, K., Lebens, M., Nordstrom, I., Schon, E. \& Holmgren, J. (2001). Cholera toxin B subunit as a carrier protein promotes antigen presentation and increases CD40 and C86 expression on antigen-presenting cells. Infect Immun 69, 5716-5725.

Han, T. K. \& Dao, M. L. (2005). Differential immunogenicity of a DNA vaccine containing the Streptococcus mutans wall-associated protein A gene versus that containing a truncated derivative antigen A lacking in the hydrophobic carboxyterminal region. DNA Cell Biol 24, 574-581.

Han, T. K., Yoder, S., Cao, C., Ugen, K. E. \& Dao, M. L. (2001). Expression of Streptococcus mutans wall-associated protein A gene in Chinese hamster ovary cells: prospect for a dental caries DNA vaccine. DNA Cell Biol 20, 595-601.

Jia, R., Guo, J. H., Fan, M. W., Bian, Z., Chen, Z., Fan, B., Yu, F. \& Xu, Q. A. (2006). Immunogenicity of CTLA4 fusion anti-caries DNA vaccine in rabbits and monkeys. Vaccine 24, 5192-5200.

Klavinskis, L. S., Gao, L., Barnfield, C., Lehner, T. \& Parker, S. (1997). Mucosal immunization with DNA-liposome complexes. Vaccine 15, 818-820.

Klavinskis, L. S., Barnfield, C., Gao, L. \& Parker, S. (1999). Intranasal immunization with plasmid DNA-lipid complexes elicits mucosal immunity in the female genital and rectal tracts. J Immunol 162, 254-262.

Letvin, N. L., Montefiori, D. C., Yasutomi, Y., Perry, H. C., Davies, M. E., Lekutis, C., Alroy, M., Freed, D. L., Lord, C. I. \& other authors (1997). Potent, protective anti-HIV immune responses generated by bimodal HIV envelope DNA plus protein vaccination. Proc Natl Acad Sci U S A 94, 9378-9383.

Lowe, D. B., Shearer, M. H. \& Kennedy, R. C. (2006). DNA vaccines: successes and limitations in cancer and infectious disease. J Cell Biochem 98, 235-242.

Ochiya, T., Takahama, Y., Baba-Toriyama, H., Tsukamoto, M., Yasuda, Y., Kikuchi, H. \& Terada, M. (1999). Evaluation of cationic liposome suitable for transfer into pregnant animals. Biochem Biophys Res Commun 258, 328-365.

Okada, E., Sasaki, S., Ishii, N., Aoki, I., Yasuda, T., Nichioka, K., Fukushima, J., Miyazaki, J.-I., Wahren, B. \& Okuda, K. (1997). Intranasal immunization of a DNA vaccine with IL-12 and 
granulocyte-macrophage colony-stimulating factor (GM-CSF)-expressing plasmids in liposomes induces strong mucosal and cell-mediated immune responses against HIV-1 antigens. J Immunol 159, 3638-3647.

Perrie, Y., Frederik, P. M. \& Gregoriadis, G. (2001). Liposomemediated DNA vaccination: the effect of vesicle composition. Vaccine 19, 3301-3310.

Qian, H. \& Dao, M. L. (1993). Inactivation of the Streptococcus mutans wall-associated protein A gene (wapA) results in a decrease in sucrose dependent adherence and aggregation. Infect Immun 61, 5021-5028.

Ramsay, A. J. \& Kohonen-Corish, M. (1993). Interleukin-5 expressed by a recombinant virus vector enhances specific mucosal IgA responses in vivo. Eur J Immunol 23, 3141-3145.

Robinson, H. L., Montefiori, D. C., Johnson, R. P., Manson, K. H., Kalish, M. L., Lifson, J. D., Rizvi, T. A., Lu, S., Hu, S. L. \& other authors (1999). Neutralizing antibody-independent containment of immunodeficiency virus challenges by DNA priming and recombinant pox virus booster immunizations. Nat Med 5, 526-534.

Rugg-gunn, A. (2001). Preventing the preventable - the enigma of dental caries. Br Dent J 191, 478-488.
Shiver, J. W., Davies, M.-E., Perry, H. C., Freed, D. C. \& Liu, M. A. (1996). Humoral and cellular immunities elicited by HIV-1 DNA vaccination. J Pharm Sci 85, 1317-1324.

Whiley, R. A., Russell, R. R. B., Hardie, J. M. \& Beighton, D. (1988). Streptococcus downei sp. nov. for strains previously described as Streptococcus mutans serotype h. Int J Syst Bacteriol 38, 25-29.

Whittle, B. L., Smith, R. M., Matthaei, K. I., Young, I. G. \& Verma, N. K. (1997). Enhancement of the specific mucosal IgA response in vivo by interleukin-5 expressed by an attenuated strain of Salmonella serotype Dublin. J Med Microbiol 46, 1029-1038.

Wu, H. Y. \& Russell, M. W. (1993). Induction of mucosal immunity by intranasal application of a surface protein with the cholera toxin $\mathrm{B}$ subunit. Infect Immun 61, 314-322.

Yasuda, Y., Matano, K., Asai, T. \& Tochikubo, K. (1998). Affinity purification of recombinant cholera toxin B subunit oligomer expressed in Bacillus brevis for potential human use as a mucosal adjuvant. FEMS Immunol Med Microbiol 20, 311-318.

Yoder, S. C., Cao, C., Ugen, K. E. \& Dao, M. L. (2000). High-level expression of a truncated wall-associated protein A from the dental cariogenic Streptococcus mutans. DNA Cell Biol 19, 401-408. 